\title{
Junkyard PCR
}

Researchers have combined basic principles of fluid dynamics with surprisingly simple building materials to assemble a cheap and effective PCR system.

Remember when a thermocycler was the most expensive equipment in your lab? This may no longer be the case, but even so, the idea of building your own PCR equipment out of a rubber sheet, a glass slide and a bit of wire may sound like something from an episode of 'MacGyver'. Nonetheless, a pair of German scientists have done just that, proving that reality can still occasionally get the edge on fiction.

The idea of using convection, the process of fluid circulation in a closed system in response to heat, as the basis for PCR reactions first emerged in 2002, when two research groups independently conceived model convective PCR systems (Braun \& Libchaber, 2002; Krishnan et al., 2002).
In such systems, DNA in solution near a heating element is denatured, and the heated mix flows to a cooler part of the chamber where annealing can take place, after which the annealed DNA is displaced back toward the heating element by the continuing fluid movement, creating conditions for primer extension, before returning to denaturing temperatures yet again.

Convection PCR pioneer Dieter Braun, of the Ludwig Maximilians Universität München, built his first such apparatus with an infrared laser to generate heat. "It was kind of an accidental finding when I was a post-doc at Rockefeller University" he says. "I wanted to have infrared lasers with which I could heat water... just for making very fast temperature oscillations in liquid, and the first experiment we switched on the laser and saw this very

DNA CLONING AND AMPLIFICATION

\section{CLONING KEEPS ON ROLLING}

\begin{abstract}
A small but valuable change to the procedure for rolling-circle amplification creates a useful cellfree cloning method.

Despite attempts to develop faster alternatives for cloning and amplification of DNA, the reliability of Escherichia coli-based methods still makes them the techniques of choice. There are situations, however, when these methods just don't work well. Clyde Hutchison and colleagues ran into one of these situations at the J. Craig Venter Institute. "We had some pieces of DNA that we weren't able to clone in $E$. coli and this got us thinking about this problem of cloning things without cells," remarks Hutchison. This thinking soon culminated in
\end{abstract}

a method of cell-free cloning using \$29 DNA polymerase (Hutchison et al., 2005).

The authors were familiar with this polymerase because of their previous work generating a synthetic genome of the $\phi \mathrm{X} 174$ bacteriophage. It seemed logical that the $\phi 29$ rolling circle amplification method of Lasken and colleagues (Dean et al., 2001) should allow reliable cellfree cloning. Hutchison remarks, "We thought we should be able to do single-molecule amplification, but it turned out to be rather difficult due to DNA synthesis in the absence of added template." They found they could overcome this problem, however, by drastically reducing the reaction volume. This change allowed 
fast convection flow, and at that time other people in the lab were working with PCR, and we thought that this could possibly be combined." Having proven the concept, Braun now sought to make a simpler device, and he describes the results of his work in a new paper from Applied Physics Letters (Hennig \& Braun, 2005). "The question was, 'Can we do it more simply?," says Braun. "And the result of that is this PCR method-essentially, all you need is a hot wire that you put into your solution." This description, though modest, is also accurate: the PCR mix is simply put into open 'wells' formed from holes in a silicone sheet stuck to a glass cover slip, then heated by a small copper wire attached to a heating element, with mineral oil on top to prevent evaporation. Cycle parameters can be changed by adjusting the heating element. Braun's group showed rapid amplification of 100-base-pair templates, and based on his experiences with laser convection, he anticipates that larger targets should also work. "There you could amplify up to 2,000 base pairs," he says. "We set similar conditions for the wire, and it didn't appear in our hands to be much different, but we didn't test that yet... [but] we're quite optimistic that we can do it eventually."

As effective as this approach is, Braun is pragmatic about the research niche his invention will likely occupy. "We can show that we are as fast as a really fast PCR machine, but we don't have an edge compared to the conventional techniques," says Braun. "So the application of this technique would be really more for a point-of-care [setting]." But for researchers in such settings, including clinics in rural areas or in the developing world, this technology could potentially prove a godsend, making diagnostic PCR simple and-for a change-dirt cheap.

\section{Michael Eisenstein}

\section{RESEARCH PAPERS}

Hennig, M. \& Braun, D. Convective polymerase chain reaction around micro immersion heater. Appl. Phys. Lett. 87, 183901 (2005).

Braun, D. \& Libchaber, A. Trapping of DNA by thermophoretic depletion and convection. Phys. Rev. Lett. 89, 188103 (2002).

Krishnan, M. et al. PCR in a Rayleigh-Bénard convection cell. Science 298, 793 (2002). them to cleanly amplify single DNA molecules several kilobytes in length, culminating in cell-free cloning of double-stranded DNA.

This small but important methodological advance could be very useful for a number of different applications. One obvious application is the sequencing of bacteriophages. As Hutchison says, "Bacteriophages are loaded with genes that are lethal to $E$. coli, so sequencing them by shotgunning into $E$. coli has [posed] problems." By eliminating $E$. coli from the equation, rolling circle amplification by $\phi 29$ DNA polymerase overcomes this problem. The other potential application that has received a lot of attention recently is a new sequencing technology developed by the company 454 Life Sciences.

In '454 sequencing' the target DNA is broken into pieces, immobilized, amplified by PCR and sequenced-all without cloning. This results in a large number of short reads, which can be hard to assemble completely. Completing the assembly can be done by conventional sequencing, but the necessary preliminary cloning of the DNA fragments remains a bottleneck in the process, and if you need to get a genome sequence very fast, combining ' 454 data' with a cloningfree method of sequencing would be more rapid. According to Hutchison, "454 Life Sciences is developing other things that may make this application not so important, but I think it may still be valuable."

\section{Daniel Evanko}

RESEARCH PAPERS

Hutchison, C.A., III et al. Cell-free cloning using $\$ 29$ DNA polymerase. Proc. Natl. Acad. Sci. USA 102, 17332-17336 (2005).

Dean, F.B. et al. Rapid amplification of plasmid and phage DNA using $\$ 29$ DNA polymerase and multiply primed rolling circle amplification. Genome Res. 11, 1095-1099 (2001). 